Hier können Sie CME-Punkte sammeln a) für die Pflichtfortbildung aller Vertragsärzte und b) für freiwillige Fortbildungszertifikate, die viele Landesärztekammern anbieten.

Die Multiple-Choice-Fragen beziehen sich auf den vorangegangenen Fortbildungsbeitrag (Seiten 31-35). Die Antworten ergeben sich z. T. aus dem Text, z. T. beruhen sie auf medizinischem Basiswissen!

Wenn Sie 70\% der Fragen richtig beantworten, erhalten Sie 2, bei 100\% 3 CME-Punkte.

Einsendeschluss: 6. April 2008 (www.cme-punkt.de) bzw. 20. Dezember 2007 (Teilnahme per Brief).
CME-Herausgeber- und Review-Board:

Dr. H. J. K. Barwitz, Prof. Dr. A. Berghaus, Prof. Dr. Dr. h.c. Th. Brandt, Prof. Dr. W. G. Daniel, Prof. Dr. W. Eisenmenger, Prof. Dr. K. Friese, Prof. Dr. H. S. Füessl, Prof. Dr. B. Göke, Prof. Dr. R. Gradinger, Prof. Dr. Dr. h.c. A. Hofstetter, Prof. Dr. H. Holzgreve, Prof. Dr. A. Imdahl, Prof. Dr. K. Krüger, Prof. Dr. H.-J. Möller, Prof. Dr. Dr. h.c. G. Plewig, Prof. Dr. D. Reinhardt, U. Weigeldt.

\title{
Fragen zum Thema „Berufsbedingte Erkrankungen der Haut“
}

- 1. Welche Aussage trifft nicht zu?

A Bei Arbeitsunfällen können die ärztlichen Leistungen von Anfang der Behandlung an zulasten des UVTr durchgeführt werden.

B Eine Unfallanzeige/Berufskrankheitenanzeige kann auch durch den Arbeitgeber erstattet werden.

C Wenn ein Behandlungsauftrag durch den UVTr erfolgt, können notwendige Arzneimittel zulasten des UVTr verordnet werden, ohne dass dadurch das Arzneimittelbudget belastet wird.

D Wenn ein Behandlungsauftrag durch den UVTr erfolgt, können u. U. nicht rezeptpflichtige Arzneimittel oder Basistherapie zulasten des UVTr verordnet werden.

E Bei jeder Berufsdermatose besteht für den Versicherten Anspruch auf Entschädigung durch den UVTr.

\section{Welche Aussage ist falsch?}

A Was Berufskrankheiten im Sinne des Gesetzes sind, geht aus der Berufskrankheitenverordnung hervor.

B Bei erhöhter Infektionsgefahr kann bei versicherten Personen auch eine im Rahmen der beruflichen Tätigkeit erworbene sexuell übertragbare Erkrankung zulasten des UVTr behandelt werden.

C UV-bedingte Karzinome der Haut werden unter der BK-Nr. 0815 gemeldet.

D Die BK-Nr. 5101 fasst ätiologisch unterschiedliche Erkrankungen zusammen.

E Die Verschlimmerung einer Psoriasis kann u. U. eine BK darstellen.

\section{Welche Aussage trifft nicht zu?}

A Unter der Anfangsziffer 1 werden Erkrankungen durch unterschiedliche toxische Stoffe gemeldet.
B Arsenkeratosen können eine Indikatorfunktion für maligne Karzinome an inneren Organen darstellen.

C Chlorakne wird durch halogenierte Kohlenwasserstoffe (Dioxine) ausgelöst.

D Bei dem geringsten Verdacht auf eine Berufsdermatose soll unverzüglich eine Umschulung eingeleitet werden.

E Bei Naturlatexallergie kann es zur Kontakturtikaria kommen, es können aber weiterreichende Symptome bis hin zum anaphylaktischen Schock (Kontaktanaphylaxie) auftreten.

- 4. Welche Aussage zur Prävention trifft nicht zu?

A In den Technischen Regeln für Gefahrstoffe wird der Umgang mit schädlichen Stoffen am Arbeitsplatz geregelt.

B Betriebsärzte tragen für die Primärprävention am Arbeitsplatz Sorge.

C Eine Krankschreibung ist nach Abheilung des Hautbefunds auch dann möglich, wenn am Arbeitsplatz für Allergenkarenz nicht gesorgt ist.

D Schutzhandschuhe schützen zuverlässig vor Handekzemen.

E Der Arbeitgeber muss ggf. geeignete Schutzkleidung oder Arbeitshandschuhe zur Verfügung stellen.

- 5. Welche Situation ist riskant für die Ausbildung eines kumulativ-toxischen Kontaktekzems der Hände?

A Arbeiten in der Kälte

B Arbeiten in der Hitze

C Arbeiten im feuchten Milieu mit Schutzhandschuhen

D Umgang mit Papier

E Arbeiten im Freien
- 6. Teer- oder Pechwarzen sind Präkanzerosen. Nach welcher Latenzzeit ist frühestens mit Karzinomen zu rechnen?

A 6 Monaten B 1-2 Jahren

C 3-4 Jahren D 8-10 Jahren

E > 25 Jahren

- 7. Als „wiederholt rückfällig“ gilt eine Erkrankung nach der Berufskrankheitenverordnung, wenn

A mehr als fünf Krankheitsschübe auftreten.

B es zu mehr als zehn Schüben kommt.

C zwischen den einzelnen Krankheitsschüben nicht mehr als sechs Monate liegen.

D mindestens 3 Krankheitsschübe, d.h. Ersterkrankung und 2 Rückfälle auftreten.

E drei Krankheitsschübe im Lauf eines Jahres auftreten.

- 8. In welcher Körperregion tritt das kumulativ-toxische Kontaktekzem am häufigsten auf?

A Gesicht $\quad$ B Hände $\quad$ C Gesäß

D Füße E Behaarter Kopf

- 9. Wodurch wird die allergische Kontakturtikaria ausgelöst?

A T-Lymphozyten B B-Lymphozyten

C IgM-Antikörper D Interleukin-6

E Spezifische IgE-Antikörper

- 10. Unter welchen Umständen muss eine Überweisung zum D-Arzt erfolgen?

A Bei jeder beruflich bed. Hautverletzung

B Wenn die Verletzung zu einer länger als den Unfalltag andauernden Arbeitsunfähigkeit führt

C Nur bei gesetzl. versicherten Personen

D Bei Verdacht auf Fahrlässigkeit

E Bei Hinweisen auf Trunkenheit während des Unfalls

\section{ANTWORTFORMULAR}

Mit Einreichen dieses Fragebogens erkläre ich mich damit einverstanden, dass die angegebenen Daten zum Zweck der Teilnahmebestätigung gespeichert und bei erfolgreicher Teilnahme an den Einheitlichen Informationsverteiler (EIV) der Ärztekammern weitergegeben werden.

Wichtiger Hinweis:

Eine Auswertung ist künftig nur noch möglich, wenn Sie Ihre EFN auf dem Antwortformular angeben! Nähere Hinweise hierzu unter: www.cme-punkt.de/faq.htm

\section{"Berufsbedingte Erkrankungen der Haut“}

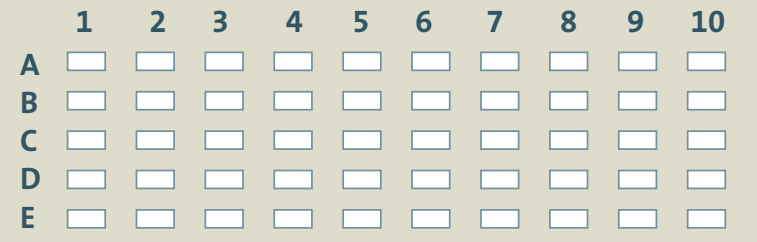

Bitte kreuzen Sie pro Frage je eine Antwort deutlich an.
Name, Vorname Fachrichtung
Und so kommen Sie zu Ihren Punkten:

Teilnahme im Internet: unter www.cme-punkt.de. Dort führen wir für Sie ein elektronisches Punktekonto.

Teilnahme per Brief: Fragebogen ausfüllen und mit frankiertem Rückumschlag an: Urban \& Vogel GmbH CME MMW-Fortschr. Med. Postfach 81664 München. 